\title{
Resolving Individual and Abstract Anaphora in Texts and Dialogues
}

\author{
Costanza Navarretta \\ Center for Sprogteknologi, University of Copenhagen \\ Njalsgade 80 \\ 2300 Copenhagen S \\ costanza@cst.dk
}

\begin{abstract}
This paper describes an extension of the DAR-algorithm (Navarretta, 2004) for resolving intersentential pronominal anaphors referring to individual and abstract entities in texts and dialogues. In DAR individual entities are resolved combining models which identify high degree of salience with high degree of givenness (topicality) of entities in the hearer's cognitive model, e.g. (Grosz et al., 1995), with Hajičová et al.'s (1990) salience account which assigns the highest degree of salience to entities in the focal part of an utterance in Information Structure terms, which often introduce new information in discourse. Anaphors referring to abstract entities are resolved with an extension of the algorithm presented by Eckert and Strube (2000). The extended DAR-algorithm accounts for differences in the resolution mechanisms of different types of Danish pronouns. Manual tests of the algorithm show that DAR performs better than other resolution algorithms on the same data.
\end{abstract}

\section{Introduction}

Although most pronominal anaphora resolution algorithms only account for anaphors referring to individual entities, anaphors referring to abstract entities evoked by verbal phrases, clauses or discourse segments (henceforth APAs) are quite common in English see i.a. (Byron and Allen, 1998) and even more in Danish (Navarretta, 2004). Recently, two algorithms for resolving APAs and individual pronominal anaphors (henceforth IPAs) in specific English dialogues have been proposed Eckert and Strube's (2000) ES00 and Byron's (2002) PHORA. Furthermore Strube and Müller (Strube and Müller, 2003) have presented a machine learning approach for resolving APAs in spoken dialogues. ${ }^{1}$ Both ES00 and PHORA \footnotetext{
per.
}

recognise IPAs and APAs on the basis of semantic constraints on the argument position occupied by the anaphors and account for differences in reference between personal and demonstrative pronouns. In ES00 demonstrative pronouns preferentially refer to abstract entities, while personal pronouns preferentially refer to individual ones. ES00 resolves IPAs applying Strube's (1998) algorithm. In PHORA the antecedents of personal pronouns are searched for looking at their degree of salience which is implemented by word order as in (Grosz et al., 1995). Demonstratives, instead, are searched for in the list of activated entities (Gundel et al., 1993) containing non NP antecedents, which are assumed to be less salient. In PHORA demonstratives can also refer to Kinds.

ES00 requires that the structure of dialogues has been marked. Byron's PHORA-algorithm does not rely on predefined dialogue structure, but only searches for abstract antecedents of APAs in the sentence preceding the anaphor. Thus it does not account for APAs referring to larger discourse segments. PHORA relies on both semantic knowledge and a model of speech acts and accounts for more phenomena than ES00. Differing from ES00, PHORA has been implemented.

To resolve IPAs and APAs in Danish texts and dialogues Navarretta (2004) has proposed the so-called DAR-algorithm (Navarretta, 2004). In DAR APAs are resolved following the ES00 strategy, but DAR accounts for the Danish abstract anaphors which occur in much more contexts than the English ones. Individual anaphors are resolved in DAR following a novel strategy which combines models which identify high degree of salience with high degree of givenness (topicality) of entities in the hearer's cognitive model, e.g. (Grosz et al., 1995), with Hajičová et al.'s (1990) salience account which assigns the highest degree of salience to entities in the focal part of an utterance in Information Structure 
terms. These entities often introduce new information in discourse.

In the present paper we describe an extended version of the DAR-algorithm accounting for differences in the reference of various types of Danish demonstratives which we found analysing the uses of pronouns in three text collections (computer manuals, novels and newspaper articles) and three corpora of recorded naturallyoccurring dialogues (the SL (Duncker and Hermann, 1996), the BYSOC (Gregersen and Pedersen, 1991) and the PID corpora (Jensen, 1989)). In the following we first discuss the background for our proposal (section 2) then we describe the extended DAR-algorithm (section 3). In section 4 we evaluate it and compare its performance with that of other known algorithms. Finally we make some concluding remarks.

\section{Background for DAR}

In most applied approaches resolving pronominal anaphors mainly consists in the following steps: 1: determining the anaphoric antecedent domain; 2: choosing the most prominent or salient antecedent among possible candidates. Thus determining the degree of salience of discourse elements, henceforth DEs, is essential to anaphor resolution. Although there is not always an identity relation between linguistic antecedents and referents, we also follow this strategy, well aware that it is particularly problematic for resolving APAs (se especially (Webber, 1991)). Nearly all salience-based algorithms identify high degree of salience with high degree of givenness of DEs. In fact, although different criteria are used for ranking DEs, such as linear order, hierarchy of grammatical roles, information structure, Prince's Familiarity Scale (Prince, 1981), all algorithms assign the highest prominence to the DEs which are most topical, known, bound, familiar and thus given, e.g. (Grosz et al., 1995; Brennan et al., 1987; Strube, 1998). Analysing the Danish data we found a restricted number of cases where high degree of salience did not correspond to high degree of topical ity, as it is the case in example (1). (1) A: hvem...hvem arbejdede $[\text { din mor }]_{i}$ med? (with whom... whom did [your mother $]_{i}$ work) B: $[\text { Hun }]_{i}$ arbejdede med [vores nabo $]_{k}$ ([She $]_{i}$ worked with [our neighbour $]_{k}$ ) $[\text { Hun }]_{k}$ var enke ... havde tre sønner [BYSOC] ([She $]_{k}$ was a widow... had three sons) In (1) the antecedent of the second occurrence of the pronoun hun (she) is the object vores nabo (our neighbour) which provides the information requested in the preceding question. This nominal is assigned lower prominence than the subject pronoun hun (she) in most salience models.

The only salience model which departs from the givenness ${ }^{2}$ assumption has been proposed by Hajičová et al. (1990). Hajičová et al., in fact, assign the highest degree of salience to DEs in the focal part of an utterance in information structure terms (Sgall et al., 1986). These entities often represent new information. Hajičová et al.'s approach is original and can account for the data in (1), but it is problematic from an applied point of view. In the first place it is difficult to determine the information structure of all utterances. Secondly, focal candidate antecedents are ranked highest in Hajičová et al.'s model, but they still compete with given candidate antecedents in their system. Finally the data does not confirm that all entities in the focal part of an utterance have the highest degree of accessibility.

We agree with Hajičová's insight, but in order to operationalise the influence of focality in a reliable way propose the following. Accessibility by default is connected with givenness as assumed in most algorithms. However, when speakers explicitly change the degree of accessibility of entities in discourse by marking them as salient with information structure related devices, these entities get the highest degree of salience and are proposed as the preferred antecedents of anaphors. In cases of explicit focus marking the shift of focus of attention is as coherent as continuing speaking about the same entities, because it is preannounced to the addressee. On the basis of the data we propose a list of identifiable constructions in which explicit focus marking occurs. ${ }^{3}$ Examples from the list are the following:

a: Entities referred to by NPs which in Danish are focally marked structurally (clefts, existential and topicalised constructions).

b: Entities referred to by NPs that follow focusing adverbs.

c: Entities focally marked by the prosody (if this information is available) and/or entities providing information requested in questions, as in (1).

\footnotetext{
${ }^{2}$ Giveness subsumes here concepts such as topicality and familiarity.

${ }^{3}$ Many of these constructions are also studied in the Information Structure and in some anaphora resolution literature, e.g. (Sidner, 1983).
} 
Givenness preference in Danish can be modelled by the hierarchy of verbal complements. In addition to salience preferences we found that parallelism can account for numerous uses of Danish anaphors. ${ }^{4}$ Inspired by the work of (Kameyama, 1996) we have defined a preference interaction model to be used in resolution. Our model is given in figure $1 .^{5}$ The interac-

Parallel. $\supseteq$ Focality $\supseteq$ Pronom. chain $\supseteq$ Givenness

Figure 1: Interaction of preferences

tion model states that givenness preferences are overridden by focality preference, when in conflict, and that they all are overridden by parallelism.

DAR also accounts for reference differences between Danish demonstrative and personal pronouns. Weak (cliticised and unstressed) pronouns usually refer to the most salient entity in the utterance. Strong (stressed and demonstrative) pronouns emphasise or put in contrast the entities they refer to and/or indicate that their antecedents are not the most expected ones. ${ }^{6}$ Demonstratives preferentially refer to abstract entities, while personal pronouns preferentially refer to individual entities in ambiguous contexts. All these differences are also accounted for in the literature on anaphora. However we also found more language-specific peculiarities in our data. Two examples of these pecularities are the following. The Danish demonstratives denne/dette/disse (this common gender/this neuter gender/these) never corefer with a subject antecedent intrasententially. In the few cases where they have a subject antecedent in a preceding clause, there are no other antecedent competitors. The abstract anaphor dette, furthermore, is often used to refer to the last mentioned situation in the previous sentence, often expressed in a subordinated clause, and not to the whole sentence or to an abstract anaphor in the preceding sentence. The partic-

\footnotetext{
${ }^{4}$ According to parallelism in adjacent utterances with parallel grammatical complements, the preferred antecedent of an anaphor in the second utterance is the linguistic expression in the first utterance with the same grammatical function.

${ }^{5}$ Commonsense preferences which override all the other preferences are not implemented.

${ }^{6}$ The most frequent Danish third person singular gender pronoun det can both be a personal pronoun (corresponding to it) and a demonstrative pronoun (corresponding to this/that). In the latter case it is always stressed.
}

ular phenomena are also accounted for in DAR.

Approx. half of the APA occurrences in our dialogues refer to entities evoked by larger discourse segments (more turn takings). Thus we follow Eckert and Strube's approach of marking the structure of dialogues and searching for APA antecedents in the right frontier of the discourse tree (Webber, 1991). DAR presupposes different discourse structures for texts and dialogues. DAR follows the ESO0 and PHORA strategy of discriminating between IPAs and APAs by rules looking at the semantic constraints on the predication contexts in which the anaphors occur. DAR relies on more discriminating rules than ES00, which were defined on the basis of large amounts of data and of the encodings of a large computational lexicon.

DAR uses language-specific rules to account for Danish APAs. These occur in much more contexts than in English where elliptical constructions or other anaphors such as too and so are used. Examples of Danish-specific uses of abstract anaphors are given in (2)-(3).

(2) Han var sulten. Det var jeg ikke. [PID]

(lit. He was hungry. That was I not)

(My friends were hungry. I wasn't.)

(3) Han kunne svømme, men det kunne hun ikke

(lit. He could swim, but it could she not)

(He could swim, but she couldn't)

A language-specific rule recognising APAs is the following: constructions with modal verbs and an object, such as $x$ skal man (lit. $x$ shall one) (one shall), $x$ vil man (lit. $x$ will one) (one will).

\section{The DAR Algorithm}

\subsection{Search Space and DE lists}

DAR presupposes the discourse structure described by Grosz and Sidner (1986). The minimal discourse unit is the utterance $U$. Paragraphs correspond to discourse segments in texts. In dialogues discourse segments were manually marked (se section 4). The dialogues were structured with Synchronising Units $(S U)$ according to the definitions in ES00.

The immediate antecedent search space of a pronoun $x$ in utterance $U_{n}$ is the previous utterance, $U_{n-1}$. If $U_{n}$ is the first component in $S U_{m}$ in dialogues the immediate search space for $x$ is $S U_{m-1}$. DAR assumes two antecedent domains depending on whether the pronoun has or has not been recognised as an IPA. The antecedent domain for IPAs is first $U_{n-1}$ and then the pre- 
ceding utterances in the right frontier of the discourse tree searched for in recency order. ${ }^{7}$ The antecedent domain for APAs or anaphors which can both be IPAs and APAs is $U_{n-1}$.

DAR operates on two lists of DEs, the Ilist and the Alist. The Ilist contains the NPs referred to in $U_{n-1}$ ranked according to their degree of salience and enriched with information on gender, number, animacy and other simple semantic types necessary to implement selectional restrictions. In the Ilist information about the grammatical role of nominals is provided and strongly focally marked elements are indicated. The leftmost element in the Ilist is the most salient one. Givenness and focality preferences are accounted for in the Ilist, as illustrated in figure 2. Focally marked entities are put in front of the list while the remaining DEs are ordered according to verbal complement order. Inside verbal complements nominals are ordered according to their occurrence order as illustrated in the second row of the figure. The abstract entities which are referred to by an APA in $U_{n-1}$ or $S U_{m-1}$ are encoded in the Alist. They are removed from the list after a new utterance ( $S U$ in dialogues) has been processed if they have not been mentioned in it. The context ranking for abstract entities is that proposed by Eckert and Strube (2000).

\subsection{The Algorithm and Its Functions}

DAR consists of two different functions RESOlveDet and REsolveIPA. The former is applied if the actual pronoun $x$ is third person singular neuter, while the latter is applied in all the remaining cases:

\section{if $x$ is singular \& neuter then go to REsolveDet $(x)$ else go to $\operatorname{ResolveIpA}(x)$}

The main steps of RESOLVEIPA are given in figure 3. The REsolveIPA approach of indicating possible reference ambiguities resembles that proposed by Kameyama (1996). The main structure of the function RESOLVEDET is inspired by ES00. ResOlveDet tests the pronoun $x$ using the IPA and APA discriminating rules discussed in section 2. RESOLVEDET is simplified in figure 4. RESOlvEIPA-NEU is like RESOLVEIPA except that it returns if no NP antecedents are found in $U_{n-1}$ (case A) so that ResolveApa can be applied. ResolveApa

\footnotetext{
${ }^{7}$ The search space in Es00 is the preceding utterance for all pronouns.
}

distinguishes between types of pronoun. If $x$ is weak, the preferred antecedent is searched for among the elements indicated in the context ranking, unless it is the object of the verb gøre (do), modals, have (have) or the abstract subject in copula constructions. In these cases the pronoun is resolved to the VP of the element in the A-list or in the context ranking. If $x$ is strong RESOLVEAPA attempts to resolve or classify it as vague depending on the type of pronoun. This part of the algorithm is specific to Danish and accounts for the fact that different strong pronouns preferentially refer to different abstract entities in the data. Resolved APAs are inserted into the Alist. In case of failure RESOlveApA returns so that RESOlveIPANEU can be applied. If both functions fail, the pronoun is classified as vague.

\subsection{Some Examples}

In the following we look at the resolution of example (1) from section 2. The simplified Ilists and Alists after each utterance has been processed are given in figure 5. (1) contains three $S U$ s. $U_{2}$ is an $\mathbf{I} / \mathbf{A}$ thus it belongs to two synchronising units $\left(S U_{1}\right.$ and $\left.S U_{2}\right)$. The Ilist after $U_{1}$ has been processed, contains one element, din mor (your mother). In $U_{2}$ the personal pronoun hun (she) occurs, thus RESOLVEIPA is applied. It resolves hun to the compatible NP in the Ilist, din mor. After $U_{2}$ has been processed the Ilist contains two elements in this order: the focal marked entity vores nabo (our neighbour) and the pronoun hun (= din mor). RESOLVEIPA resolves the occurrence of the pronoun hun (she) in $U_{3}$ to the most salient candidate NP in the Ilist, vores nabo. Here focal preference overrides pronominal chain preference. Example (4) contains the APA det.

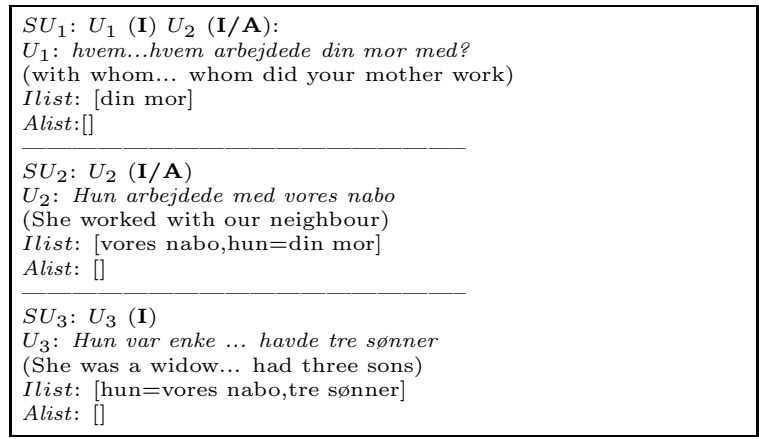

Figure 5: Ilists and Alists for example (1)

(4): Du har svart ved at se musemarkøren på skarmen. Hvordan klarer du det? [EDB] (You have difficulties seing the mouse-cursor 
Figure 2: Order of DEs in the Ilist

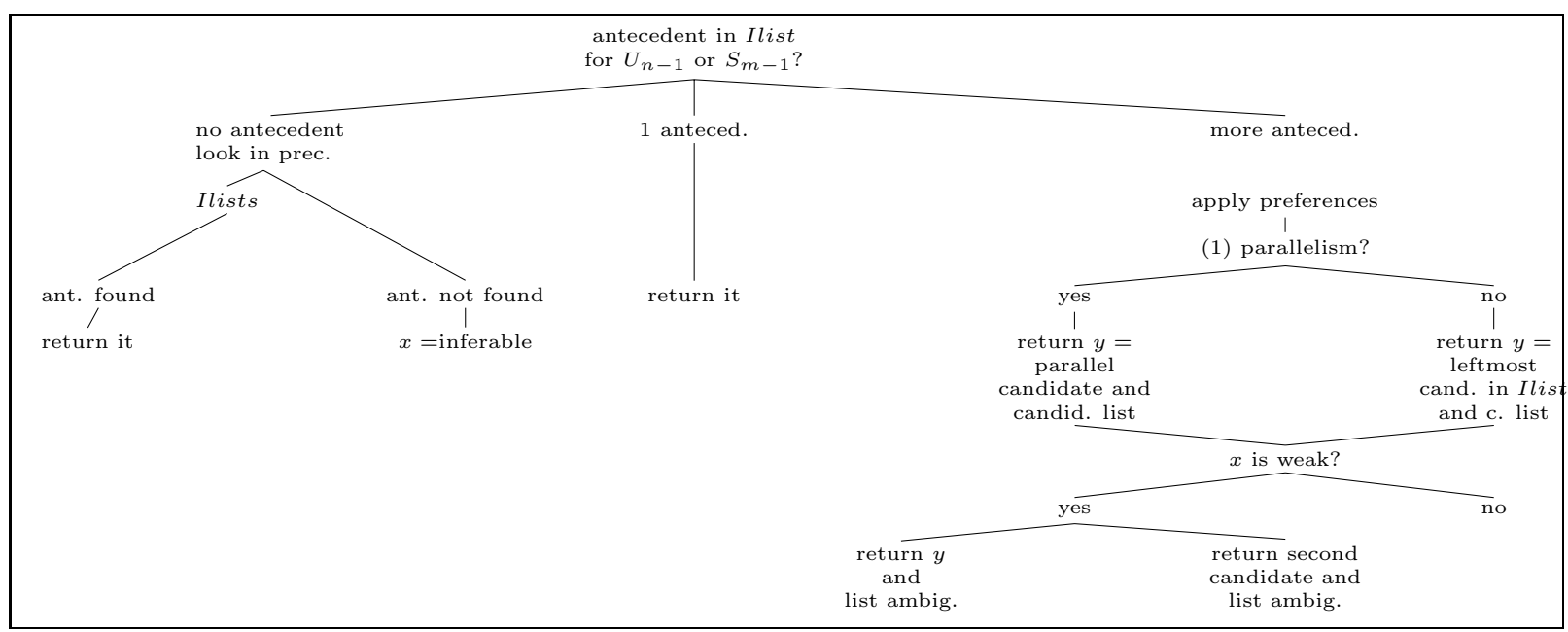

Figure 3: RESOLVEIpA

(common-gend) on the screen (common-gend). How do you manage it/this (neuter gender))? The simplified Ilists and Alists after the two utterances in (4) have been processed are presented in figure 6 . After $U_{1}$ has been pro-

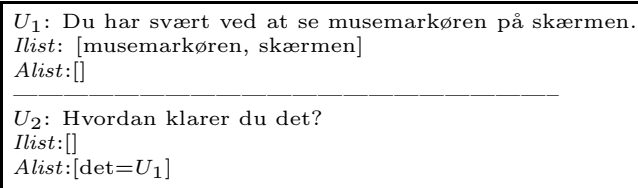

Figure 6: Ilists and Alists for example (4)

cessed there are two common gender singular NPs in the Ilist, musemarkøren (the mouse cursor) and skarmen (the screen). In $U_{2}$ the singular neuter gender pronoun det (it) occurs, thus ResolveDet is applied. The pronoun is neither IPA nor APA according to the discriminating rules. RESOLVEDET attempts to find an individual antecedent of the weak pronoun, applying the function RESOLVEIPA-NEU. RESOLVEIPA-NEU fails because the two DEs in the Ilist do not agree with the pronoun. Then the function RESOLVEAPA resolves $x$ looking at the context ranking. Being the Alist empty, $U_{1}$, is proposed as antecedent. The resolved APA is added to the Alist.

\section{Tests and Evaluation}

We have manually tested DAR on randomly chosen texts and dialogues from our collections. The performance of DAR on dialogues has been compared with that of ES00. The function for resolving IPAs (RESOLVEIPA) has similarly been tested on texts, where APAs were excluded. We have compared the obtained results with those obtained by testing BFP (Brennan et al., 1987) and STR98 (Strube, 1998). In all tests the intrasentential anaphors have been manually resolved and expletive and cataphoric uses of pronouns have been marked and excluded from the test. Dialogue act units were marked and classified by three annotators following (Eckert and Strube, 2000). The reliability for the two annotation tasks ( $\kappa$-statistics (Carletta, 1996)) was of 0.94 and 0.90 respectively. Pronominal anaphors were marked, classified and resolved by two annotators. The $\kappa$ statistics for the pronoun classification was 0.86 . In few cases (one in the texts and two in the dialogues) where the annotators did not agree upon resolution, the pronouns were marked as ambiguous and were excluded from the test. The results obtained for BFP and STR98 are given in table 1, while the results of DAR's RESOLVEIPA are given in table 2. In the tables CR stands for "correctly resolved", HR stands for "resolved by humans", RA stands for "resolved over all", $\mathbf{P}$ stands for precision and $\mathbf{R}$ stands for recall. Because DAR both classifies and resolves anaphors, both precision and recall (respect to human resolution) are given in table 2 . The results indicate that RESOLVEIPA performs significantly better than BFP and STR98 on the 


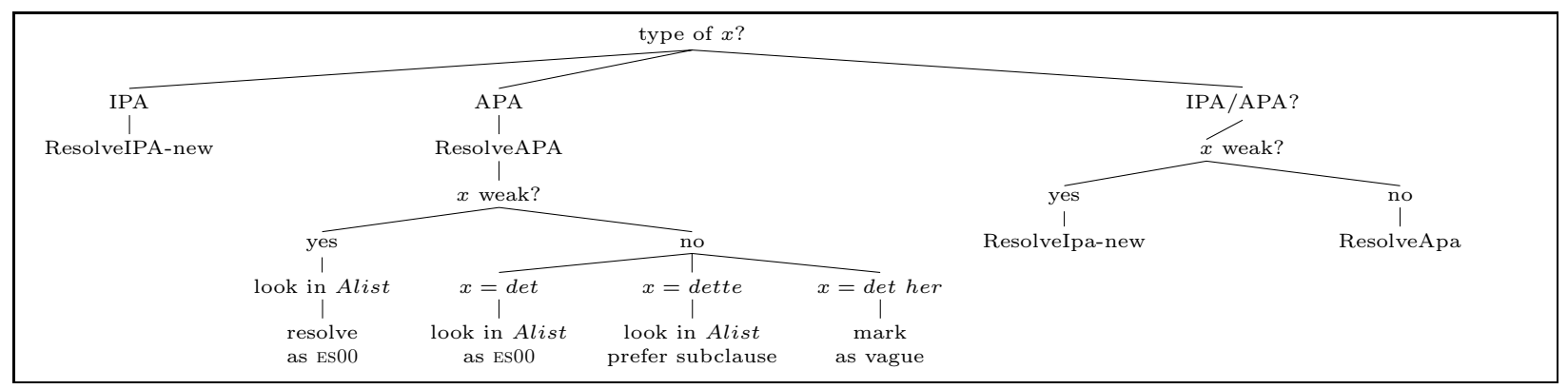

Figure 4: ResolveDet

Danish texts. The better performance of DAR was due to the account of focal and parallelism preferences, of the different reference mechanisms of personal and demonstrative pronouns and to the enlarged resolution scope. Furthermore DAR recognises some generic pronouns and inferable pronouns and excludes them from resolution, but often fails to recognise antecedentless and inferable plural pronouns, because it finds a plural nominal in the preceding discourse and proposes it as antecedent. The lack of commonsense knowledge explains many incorrectly resolved anaphors. The results of the test of

\begin{tabular}{|c|c|c|c|}
\hline algorithm & CR & HR & P \\
\hline BFP & 513 & 645 & 79.53 \\
\hline STR98 & 524 & 645 & 81.24 \\
\hline
\end{tabular}

Table 1: Results of BFP and STR98 on texts

\begin{tabular}{|c|c|c|c|c|}
\hline CR & RA & HR & P & R \\
\hline 575 & 651 & 645 & 88.33 & 89.14 \\
\hline
\end{tabular}

Table 2: Results of ResolveIpA on texts

the DAR algorithm on written texts are in table 3 . These results are good compared with the results of the function RESOLVEIpA (table 2). The discriminating rules identify correctly IPAs and APAs in the large majority of the cases. Recognition failure often involves pronouns in contexts which are not covered by the discriminating rules. In particular DAR fails to resolve singular neuter gender pronouns with distant antecedents and to identify vague anaphors, because it always "finds" an antecedent in the context ranking. Correct resolution in these cases requires a deep analysis of the context. The results of applying DAR and ES00 on Danish dialogues are reported in table $4 .^{8}$ The results of the tests indicate that DAR resolves IPAs significantly better than ES00 (which uses STR98).

\footnotetext{
${ }^{8}$ We extended ES00 with the Danish-specific identification rules before applying it.
}

\begin{tabular}{|c|c|c|c|c|}
\hline \multicolumn{5}{|c|}{ resolution IPA } \\
\hline CR & RA & HR & P & R \\
\hline 560 & 651 & 645 & 86.02 & 86.82 \\
\hline \multicolumn{5}{|c|}{ resolution APA } \\
\hline CR & RA & HR & P & R \\
\hline 68 & 87 & 77 & 78.16 & 88.31 \\
\hline
\end{tabular}

Table 3: Results of DAR on texts

DAR correctly resolves more Danish demonstrative pronouns than ESO0, because it accounts for language-specific particularities. In general, however, the resolution results for APAs are similar to those obtained for ES00. This is not surprising, because DAR uses the same resolution strategy on these pronouns. DAR performs better on texts than on dialogues. This reflects the more complex nature of dialogues. The results indicate that the IPA/APA discriminating rules also work well on dialogues. The cases of resolution failure were the same as for the texts. As an experiment we applied DAR on the

\begin{tabular}{|c|c|c|c|c|c|}
\hline \multicolumn{7}{|c|}{ resolution IPA } \\
\hline Algorithm & CR & RA & HR & P & R \\
\hline ES00 & 258 & 411 & 414 & 62.77 & 62.31 \\
\hline DAR & 289 & 386 & 414 & 74.87 & 68.81 \\
\hline \multicolumn{7}{|c|}{ resolution APA } \\
\hline Algorithm & CR & RA & HR & P & R \\
\hline ES00 & 179 & 286 & 269 & 62.59 & 66.54 \\
\hline DAR & 199 & 277 & 269 & 71.84 & 73.98 \\
\hline
\end{tabular}

Table 4: Results of ES00 and DAR on dialogues

dialogues without relying on the predefined dialogue structure. In this test the recognition of IPAs and APAs was still good, however the success rate for IPAs was of $60.1 \%$ and for APAs was of only $39.3 \%$. Many errors were due to the fact that antecedents were searched for in the preceding discourse in linear order and that ungrounded utterances were included in the discourse model. 


\section{Concluding Remarks}

In this paper we presented DAR, an algorithm for resolving IPAs and APAs in Danish texts and dialogues. In DAR differences between the referential characteristics of Danish weak and strong pronouns are accounted for and a novel strategy for resolving individual anaphors is proposed. This strategy combines givenness with focality preferences to model salience and also accounts for parallelism preferences. DAR performs significantly better on IPAs than algorithms which only rely on givenness-based salience models.

DAR extends the ES00 strategy of classifying and resolving (some types of) APAs. The tests of DAR indicate that the ES00's approach of recognising APAs is also promising for texts and other languages than English.

DAR has not been compared with PHORA which is the only abstract anaphora algorithm implemented. We find the algorithm very interesting because it addresses many of the same phenomena, but with different strategies. It would be useful to combine some of these strategies with the approaches proposed in DAR and ES00 to improve the still problematic resolution of abstract anaphors.

\section{References}

S. F. Brennan, M. W. Friedman, and C. J. Pollard. 1987. A Centering Approach to Pronouns. In Proceedings of the ACL'87, pages 155-162, CA.

D. Byron and J. Allen. 1998. Resolving demonstrative pronouns in the trains93 corpus. In Proceedings of DAARC 2, pages 68-81.

D. K. Byron. 2002. Resolving Pronominal Reference to Abstract Entities. In Proceedings of the ACL 2002.

J. Carletta. 1996. Assessing agreement on classification tasks. The kappa statistic. In Computational Linguistics, 22(2):249-254.

D. Duncker and J. Hermann. 1996. Patientord og lægeord - særord eller fællesord? Månedsskrift for Praktisk Lagegerning, pages 1019-1030.

M. Eckert and M. Strube. 2000. Dialogue acts, synchronising units and anaphora resolution. Journal of Semantics, 17:51-89.

F. Gregersen and I. L. Pedersen, editors. 1991. The Copenhagen study in urban sociolinguistics. Reitzel.

B. J. Grosz and C. L. Sidner. 1986. Attention,
Intentions, and the Structure of Discourse. Computational Linguistics, 12(3):175-284.

B. Grosz, A. K. Joshi, and S. Weinstein. 1995. Centering:A Framework for Modeling the Local Coherence of Discourse. Computational Linguistics, 21(2):203-225.

J. K. Gundel, N. Hedberg, and R. Zacharski. 1993. Cognitive status and the form of referring expressions in discourse. Language, 69(2):274-307.

E. Hajičová, P. Kuboň, and V. Kuboň. 1990. Hierarchy of Salience and Discourse Analysis and Production. In H. Karlgren, editor, Proceedings of COLING'90, volume III, pages 144-148, Helsinki.

K. A. Jensen. 1989. Projekt invandrerdansk. Technical report, Copenhagen University.

M. Kameyama. 1996. Indefeasible Semantics and Defeasible Pragmatics. In M. Kanazawa, C. Piñon, and H. de Stwart, editors, Quantifiers, Deduction and Context, pages 111-138. CSLI, Stanford, CA.

C. Navarretta. 2002. Combining Information Structure and Centering-based Models of Salience for Resolving Intersentential Pronominal Anaphora. In A. Branco, T. McEnery, and R.Mitkov, editors, Proceedings of the 4th DAARC, pages 135-140. Ediçoes Colibri.

C. Navarretta. 2004. . In Proceedings of Reference Resolution and Its Applications Workshop at ACL 2004, Barcelona, Spain.

E. F. Prince. 1981. Toward a taxonomy of given-new information. In P. Cole, editor, Radical Pragmatics, Academic Press, pages 223-255.

C. Sidner. 1983. Focusing in the Comprehension of Definite Anaphora. In M. Brady and R. Berwick, editors, Computational Models of Discourse, MIT Press, pages 267-330.

P. Sgall, E. Hajičová, and J. Panevová. 1986. The Meaning of the Sentence in its Semantic and Pragmatic Aspects. Reidel, Dordrecht.

M. Strube. 1998. Never Look Back: An Alternative to Centering. In Proceedings of COLING-ACL'98, II, pages 1251-1257.

M. Strube and C. Müller. 2003. A machine learning approach to pronoun resolution in spoken dialogue. In Proceedings of the $A C L$ '03, pages $168-175$.

B. L. Webber. 1991. Structure and Ostension in the Interpretation of Discourse Deixis. Natural Language and Cognitive Processes, 6(2):107-135, January. 